\title{
Microarray Analysis of Transcriptome of Medulla Identifies Potential Biomarkers for Parkinson's Disease
}

\author{
Xiao-Yang Liao, ${ }^{1}$ Wei-Wen Wang, ${ }^{2}$ Zheng-Hui Yang, ${ }^{2}$ Jun Wang, ${ }^{2}$ Hang Lin, \\ Qing-Song Wang, ${ }^{2} \mathrm{Yu}-\mathrm{Xian} \mathrm{Wu}{ }^{2}$ and $\mathrm{Yu}$ Liu $^{2}$ \\ ${ }^{1}$ Unit of General Practice, West China Hospital of Sichuan University, Chengdu 610041, China \\ ${ }^{2}$ Department of Neurology, Cheng Du Military General Hospital, Chengdu 610083, China
}

Correspondence should be addressed to Wei-Wen Wang; weiwenwang32@hotmail.com

Received 13 August 2013; Accepted 30 September 2013

Academic Editor: Bo Wang

Copyright (c) 2013 Xiao-Yang Liao et al. This is an open access article distributed under the Creative Commons Attribution License, which permits unrestricted use, distribution, and reproduction in any medium, provided the original work is properly cited.

\begin{abstract}
To complement the molecular pathways contributing to Parkinson's disease (PD) and identify potential biomarkers, gene expression profiles of two regions of the medulla were compared between PD patients and control. GSE19587 containing two groups of gene expression profiles [ 6 dorsal motor nucleus of the vagus (DMNV) samples from PD patients and 5 from controls, 6 inferior olivary nucleus (ION) samples from PD patients and 5 from controls] was downloaded from Gene Expression Omnibus. As a result, a total of 1569 and 1647 differentially expressed genes (DEGs) were, respectively, screened in DMNV and ION with limma package of $R$. The functional enrichment analysis by DAVID server (the Database for Annotation, Visualization and Integrated Discovery) indicated that the above DEGs may be involved in the following processes, such as regulation of cell proliferation, positive regulation of macromolecule metabolic process, and regulation of apoptosis. Further analysis showed that there were 365 common DEGs presented in both regions (DMNV and ION), which may be further regulated by eight clusters of microRNAs retrieved with WebGestalt. The genes in the common DEGs-miRNAs regulatory network were enriched in regulation of apoptosis process via DAVID analysis. These findings could not only advance the understandings about the pathogenesis of PD, but also suggest potential biomarkers for this disease.
\end{abstract}

\section{Introduction}

Parkinson's disease (PD) is the second most common neurodegenerative disorder in human, which is characterized by progressive death of dopamine-generating cells in the substantia nigra and accumulation of intraneuronal Lewy bodies containing misfolded fibrillar $\alpha$-synuclein (SNCA), which eventually results in progressive movement disorders, including shaking, rigidity, bradykinesia, and gait disturbance [1]. Epidemiologic studies have identified environmental factors such as trauma [2] and pesticide exposure [3, 4] as risk factors for $\mathrm{PD}$, while the increasing evidence demonstrates that genetic factors play significant roles in PD. Several genes have been linked to $\mathrm{PD}$, such as SNCA, leucine-rich repeat kinase 2 (LRRK2), parkin (PARK2), PTEN-induced kinase 1 (PINK1), and DJ-1 (PARK7) $[5,6]$. In addition, as an important regulator at posttranscriptional level, several miRNAs have been discovered to be involved in $\mathrm{PD}$ pathogenesis via regulating
PD-associated gene expression. For example, miR-7 and miR153 are recently described to regulate endogenous synuclein levels; inhibition of $\alpha$-synuclein expression by miR-7 protects against oxidative stress-mediated cell death $[7,8]$; several studies suggest that the role of LRRK2 in the pathogenesis of $\mathrm{PD}$ is mediated through the miRNA pathway [9].

Dorsal motor nucleus of the vagus (DMNV) and inferior olivary nucleus (ION) are two brainstem regions which may be damaged early in the course of PD [10, 11]. However, the molecular mechanism of these two regions is not well understood for PD. In this study, we aimed to compare the gene expression profiles of DMNV and ION from PD patients with that of controls using oligonucleotide microarray. Microarray experiments can simultaneously measure the expression levels of thousands of genes, generating huge amounts of data, [12] and have been applied to identify molecular markers of PD in several studies [13, 14]. In addition, the related miRNAs that were mapped to their target 


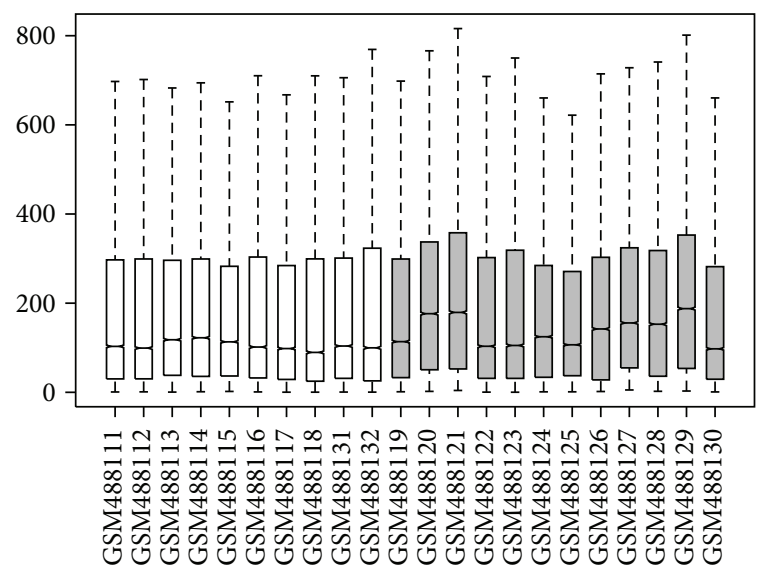

(a)

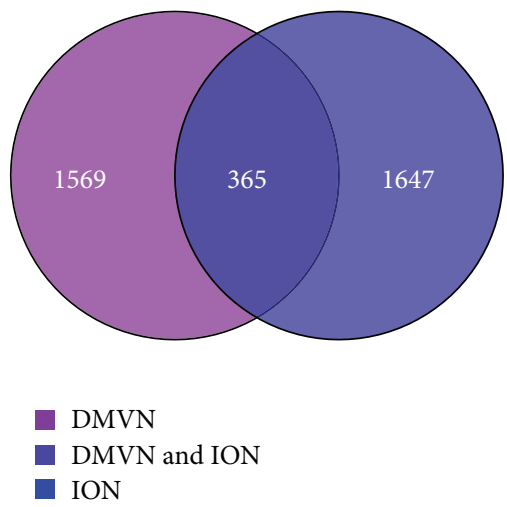

(b)

FIGURE 1: Box plot for normalized gene expression data. The medians (black lines) are almost at the same level, indicating a good performance of normalization (a). Venn diagram of differentially expressed genes identified from dorsal motor nucleus of the vagus (DMNV) and inferior olivary nucleus (ION) of PD (b).

differentially expressed genes (DEGs) were also analyzed by bioinformatics methods to reveal the regulatory mechanism.

\section{Materials and Methods}

2.1. Microarray Data. Gene expression data set GSE19587 [15] was downloaded from Gene Expression Omnibus [16]. It contained two groups of gene expression profiles: 6 DMNV samples from patients with PD and 5 from controls; 6 ION samples from patients with $\mathrm{PD}$ and 5 from controls. The platform was GPL571 [HG-U133A_2] Affymetrix Human Genome U133A 2.0 Array. Probe annotation files were also acquired.

2.2. Preprocessing and Differential Analysis. Raw data were converted into recognizable format with package affy of $R$, and missing values were then imputed [17]. After data normalization with median method [18], differential analysis between PD and control was performed using package limma [19] for DMNV and ION, respectively. |log (fold change) FC $>1$ and $P<0.05$ were set as the cut-offs to screen out DEGs.

2.3. Gene Ontology (GO) Functional Enrichment Analysis of $D E G s$. In order to identify disturbed biological functions in $\mathrm{PD}, \mathrm{GO}$ functional enrichment analysis was performed for DEGs in DMNV and ION using DAVID with a threshold of $P<0.05$ [20]. DAVID is the Database for Annotation, Visualization and Integrated Discovery, providing a comprehensive set of functional annotation tools for the investigation of the biological meaning behind large list of genes.

2.4. Comparison of DEGs between DMNV and ION. Common DEGs from the two regions of the medulla (DMNV and ION) were obtained using package Venn of $R$.

2.5. Establishing Interaction Network between Common DEGs and miRNAs and Functional Enrichment Analysis for DEGs in
Network. miRNAs which targeted the common DEGs were retrieved with WebGestalt [21, 22]. For multiple testing correction, the Benjamini-Hochberg $(\mathrm{BH})$ approach was used [23], and miRNAs with $\mathrm{BH}$-adjusted $P<0.05$ (false discovery rate $($ FDR $)<0.05)$ were selected. The regulatory network between DEGs and miRNAs and interactions between DEGs were then visualized with Cytoscape. In addition, GO functional enrichment analysis was applied on the genes in the network via DAVID with a threshold of $P<0.05$.

\section{Results}

3.1. DEGs in $D M N V$ and ION. After gene expression data normalization (Figure 1(a)), 1569 (DMNV) and 1647 (ION) DEGs for PD were screened by comparison between the samples from PD patients and controls. As shown in Figure 1(b), 385 common DEGs presented both in DMNV and ION of PD patients were extracted from these identified DEGs.

3.2. Functional Enrichment Analysis Results. Significantly overrepresented GO terms were revealed by using DAVID. A total of 24 and 28 terms were disclosed for DEGs in DMNV and ION, respectively (Figure 2), in which DEGs from DMNV and ION seemed to share similar biological processes, such as regulation of cell proliferation, positive regulation of macromolecule metabolic process, regulation of apoptosis, and so on.

3.3. miRNAs and Gene Regulatory Network. A total of 8 relevant clusters of miRNAs were retrieved with WebGestalt for the common DEGs (Table 1). Then the miRNAs-DEGs regulatory network and DEGs-DEGs interaction network were visualized with Cytoscape (Figure 3). Functional annotation was applied on the genes in the network, and 19 GO terms were revealed (Table 2), among which regulation of apoptosis was the most significant one. 

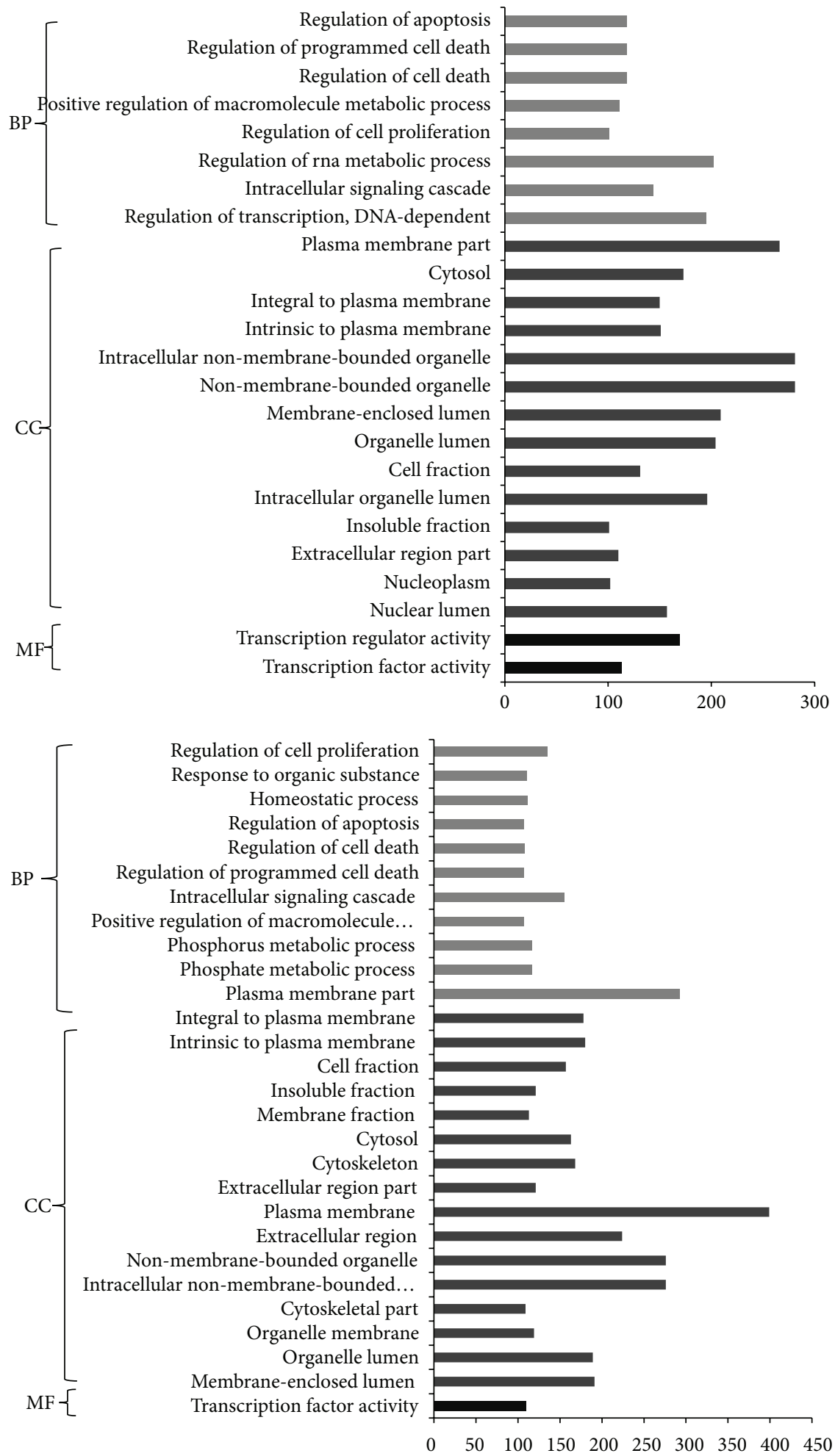

FIGURE 2: Overrepresented gene ontology terms for differentially expressed genes from dorsal motor nucleus of the vagus (DMNV, above) and inferior olivary nucleus (ION, below). BP: biological process; CC: cellular component; MF: molecular function. 


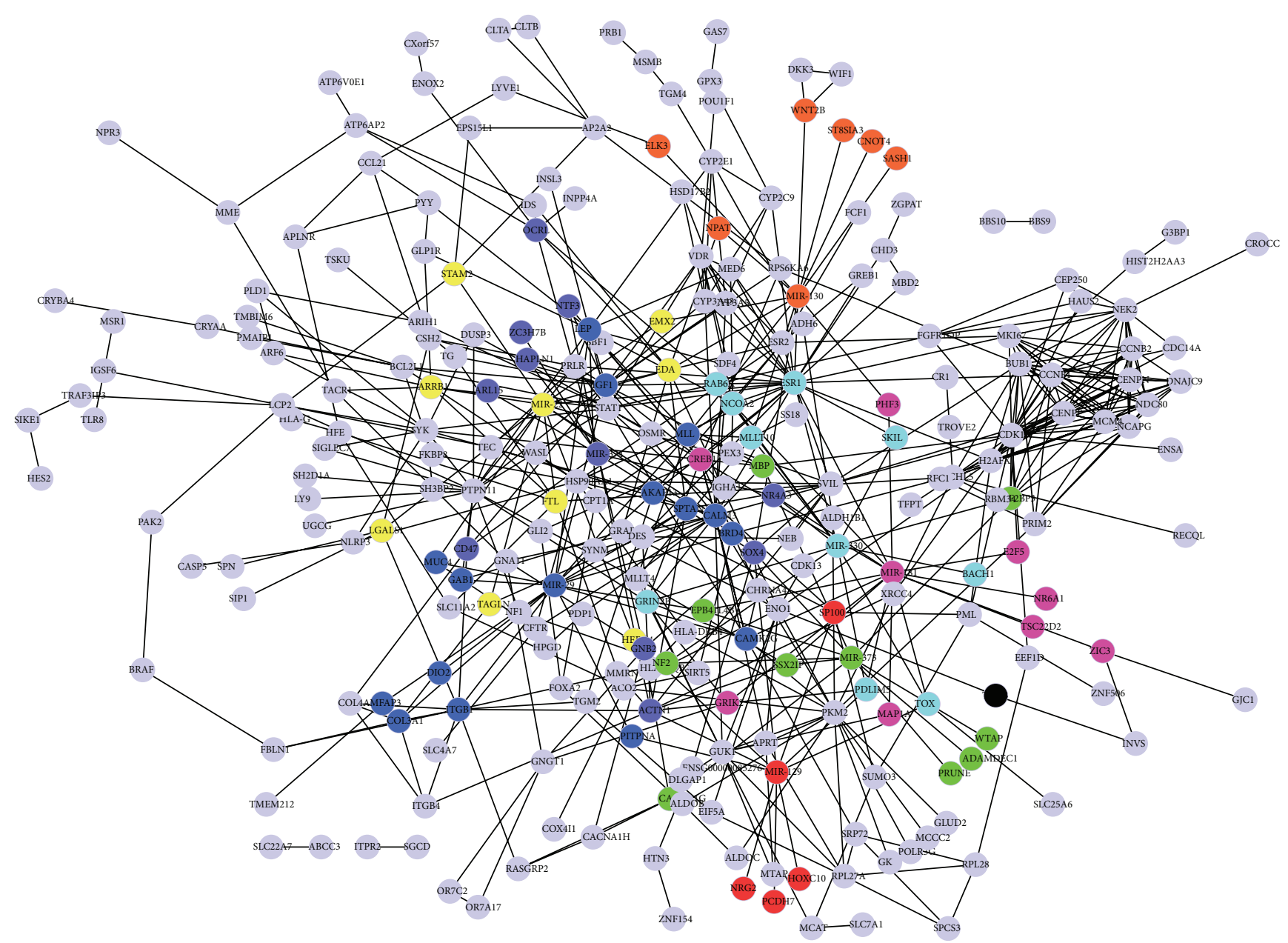

FIGURE 3: The integrated DEGs-miRNAs regulatory and DEGs-DEGs interaction network. miRNAs and their target genes shared the same color. DEGs: differentially expressed gene.

TABLE 1: Relevant miRNAs for the 365 common differentially expressed genes.

\begin{tabular}{lcc}
\hline miRNA & DB_Num & Parameters \\
\hline hsa_GGCAGCT, MIR-22 & DB_ID:780 & $O=13 ; \operatorname{raw} P=8.79 e-08 ;$ adj $P=1.76 e-06$ \\
hsa_TGAATGT, MIR-181A, MIR-181B, MIR-181C, MIR-181D & DB_ID:669 & $O=16 ; \operatorname{raw} P=3.73 e-06 ;$ adj $P=2.56 e-05$ \\
hsa_GCAAAAA, MIR-129 & DB_ID:798 & $O=10 ; \operatorname{raw} P=3.84 e-06 ;$ adj $P=2.56 e-05$ \\
hsa_TGGTGCT, MIR-29A, MIR-29B, MIR-29C & DB_ID:671 & $O=16 ; \operatorname{raw} P=9.23 e-06 ;$ adj $P=4.61 e-05$ \\
hsa_TTTTGAG, MIR-373 & DB_ID:844 & $O=10 ; \operatorname{raw} P=2.08 e-05 ;$ adj $P=8.32 e-05$ \\
hsa_TGCTTTG, MIR-330 & DB_ID:843 & $O=12 ; \operatorname{raw} P=2.76 e-05 ;$ adj $P=9.20 e-05$ \\
hsa_TTGCACT, MIR-130A, MIR-130B & DB_ID:676 & $O=13 ; \operatorname{raw} P=3.80 e-05 ;$ adj $P=0.0001$ \\
hsa_TATTATA, MIR-374 & DB_ID:727 & $O=10 ; \operatorname{raw} P=0.0002 ;$ adj $P=0.0005$ \\
\hline
\end{tabular}

DB_Num: number assigned by the database; $O$ : number of differentially expressed genes regulated by the miRNA; raw $P$ : initial $P$ value calculated according to the hypergeometric distribution; adjP: $P$ value after adjusted with the Benjamini-Hochberg correction method.

\section{Discussion}

In the present study, we identified 1569 and 1647 DEGs in DMNV and ION, respectively through the comparative analysis of transcriptome between PD and controls. Also, we found 365 common DEGs presented in both regions, as well as 8 related miRNAs which targeted these common DEGs. Finally, we constructed an integrated network, including the DEGs-DEGs interactions, and the DEGs-miRNA regulatory network consisting of 8 miRNAs (MIR-22, MIR-181, MIR-129, MIR-29, MIR-373, MIR-330, MIR-130, and MIR374) and their target common DEGs.

Apoptosis plays a critical role in the pathogenesis of $\mathrm{PD}$ $[24,25]$. In present study, many DEGs involved in apoptosis were found in the two regions of the medulla. Functional enrichment analysis of DEGs indicated that regulation of 
TABLE 2: Overrepresented GO terms in genes from the regulatory network.

\begin{tabular}{|c|c|c|}
\hline Term & Count of DEGs & $P$ value \\
\hline GO:0042981 regulation of apoptosis & 31 & 0.0026939 \\
\hline GO:0043067 regulation of programmed cell death & 31 & 0.0031183 \\
\hline GO:0010941 regulation of cell death & 31 & 0.0032783 \\
\hline GO:0070271 protein complex biogenesis & 22 & 0.0034321 \\
\hline GO:0006461 protein complex assembly & 22 & 0.0034321 \\
\hline GO:0043085 positive regulation of catalytic activity & 22 & 0.0048116 \\
\hline GO:0065003 macromolecular complex assembly & 26 & 0.0054608 \\
\hline GO:0048878 chemical homeostasis & 21 & 0.0082118 \\
\hline GO:0007267 cell-cell signaling & 23 & 0.0117819 \\
\hline GO:0043933 macromolecular complex subunit organization & 26 & 0.0119356 \\
\hline GO:0042127 regulation of cell proliferation & 28 & 0.0126938 \\
\hline GO:0042592 homeostatic process & 27 & 0.0128669 \\
\hline GO:0007049 cell cycle & 27 & 0.0185901 \\
\hline GO:0022402 cell cycle process & 21 & 0.0222268 \\
\hline GO:0006357 regulation of transcription from RNA polymerase II promoter & 25 & 0.027631 \\
\hline GO:0010604 positive regulation of macromolecule metabolic process & 28 & 0.0335407 \\
\hline GO:0031328 positive regulation of cellular biosynthetic process & 23 & 0.0437151 \\
\hline GO:0010557 positive regulation of macromolecule biosynthetic process & 22 & 0.0482295 \\
\hline GO:0009891 positive regulation of biosynthetic process & 23 & 0.049702 \\
\hline
\end{tabular}

GO: gene ontology; DEGs: differentially expressed genes.

apoptosis was the one of the top 3 biological processes for both groups of DEGs. Moreover, thirty-one DEGs in the regulatory network were also enriched in regulation of apoptosis (the top one GO term). It has been reported that some DEGs (e.g., VDR, NTF3, CREB1, and IGF1) within the apoptosis pathway may contribute to the pathogenesis of $\mathrm{PD}$ according to the previous literature. Vitamin D has been demonstrated to regulate cell proliferation in the developing brain [26], and vitamin $\mathrm{D}$ deficiency alters dopamine turnover in the forebrain and dopamine-mediated movement, resulting in high risk for $\mathrm{PD}[27,28]$. Vitamin D receptor (VDR) is the primary mediator of vitamin D's biological actions; that is, vitamin $\mathrm{D}$ is first converted to the active metabolite 1,25-dihydroxy vitamin D3. Upon binding to 1,25-dihydroxy vitamin D3, VDR is activated and interacts with vitamin D responsive elements in the promoters of vitamin $\mathrm{D}$ target genes to regulate their expression $[29,30]$. Moreover, several studies also report an association between VDR polymorphism and PD [31, 32]. Neurotrophin 3 (NTF3) is a member of the neurotrophin family, which controls the survival and differentiation of mammalian neurons. The delivery of NTFs has been postulated as a therapy for neurodegenerative disorders like PD $[33,34]$. As a member of the leucine zipper family of DNA binding proteins, CREB1 (cAMP responsive element binding protein 1) may play an important role in the dopaminergic activation of $\mathrm{c}$-fos in the striatum, and the lacking of a CREB1-induced transcription cascade may contribute to long-lasting psychomotor disorders in PD [35]. Ebert et al. report that human neural progenitor cells overexpressing IGF1 (insulin-like growth factor 1) can protect dopamine neurons and restore function in a rat model of PD [36].
miRNAs are important regulators participating in many physiological processes and thus become therapeutic targets for diseases, such as cancers and neurodegenerative diseases [37]. To discover potential molecular targets, miRNAs interacting with DEGs were retrieved in the present study and regulatory network was also constructed. Aberrant expression of miR-22 has been identified in multiple human diseases [38]. It shows low expressions in PD blood samples, and it can be used to distinguish nontreated PD from healthy subjects [39]. Ferritin light polypeptide (FTL) is regulated by miR-22. FTL is the light subunit of the ferritin protein, which is the major intracellular iron storage protein. Previous studies have indicated that disturbances in brain iron homeostasis may contribute to the pathogenesis of PD $[40,41]$. Thus, we suppose that FTL and miR-22 are worthy of further investigations to disclose their specific roles in PD. miR-181 is implicated in apoptosis. Downregulation of miR-181 permits $\mathrm{Bcl}-2$ to remain at a high level without posttranscriptional repression, which eventually leads to the gain in neuronal survival [42] and may decrease the incidence of PD. DEGs regulated by this miRNA included CREB1 and estrogen receptor 1 (ESR1). The expression level of miR-29 can also be used to distinguish nontreated PD from healthy subjects [39]. IGF1 and calcium/calmodulin-dependent protein kinase II gamma (CAMK2G) are regulated by this miRNA. CAMK2G links endoplasmic reticulum stress with Fas and mitochondrial apoptosis pathways [43]. Inhibitors of CAMK2G may be useful in preventing apoptosis in pathological settings and even treat diseases like PD.

Overall, our study provides an integrated network insight into the pathogenesis of PD and offers potential therapeutic targets for controlling the disease. Although previous studies 
have implicated that brainstem regions including DMNV and ION are relatively unaffected and not obligatory trigger sites of PD $[10,44,45]$, the genes in DMNV and ION are demonstrated to be associated with neuron death in our study, and thus deep experiment researches in these regions are still needed.

\section{References}

[1] S. Phani, J. D. Loike, and S. Przedborski, "Neurodegeneration and inflammation in Parkinson's disease," Parkinsonism and Related Disorders, vol. 18, supplement 1, pp. S207-S209, 2012.

[2] J. P. Hubble, T. Cao, R. E. S. Hassanein, J. S. Neuberger, and W. C. Koller, "Risk factors for Parkinson's disease," Neurology, vol. 43, no. 9, pp. 1693-1697, 1993.

[3] A. Ascherio, H. Chen, M. G. Weisskopf et al., "Pesticide exposure and risk for Parkinson's disease," Annals of Neurology, vol. 60, no. 2, pp. 197-203, 2006.

[4] J. M. Gorell, C. C. Johnson, B. A. Rybicki, E. L. Peterson, and R. J. Richardson, "The risk of Parkinson's disease with exposure to pesticides, farming, well water, and rural living," Neurology, vol. 50, no. 5, pp. 1346-1350, 1998.

[5] S. T. Camargos, L. O. Dornas, P. Momeni et al., "Familial Parkinsonism and early onset Parkinson's disease in a Brazilian movement disorders clinic: phenotypic characterization and frequency of SNCA, PRKN, PINK1, and LRRK2 mutations," Movement Disorders, vol. 24, no. 5, pp. 662-666, 2009.

[6] C. Y.-C. Chen, "Mechanism of BAG1 repair on Parkinson's disease-linked DJ1 mutation," Journal of Biomolecular Structure and Dynamics, vol. 30, no. 1, pp. 1-12, 2012.

[7] E. Junn, K.-W. Lee, S. J. Byeong, T. W. Chan, J.-Y. Im, and M. M. Mouradian, "Repression of $\alpha$-synuclein expression and toxicity by microRNA-7," Proceedings of the National Academy of Sciences of the United States of America, vol. 106, no. 31, pp. 13052-13057, 2009.

[8] E. Doxakis, "Post-transcriptional regulation of $\alpha$-synuclein expression by mir-7 and mir-153," Journal of Biological Chemistry, vol. 285, no. 17, pp. 12726-12734, 2010.

[9] E. Filatova, "MicroRNAs: possible role in pathogenesis of Parkinson's disease," Biochemistry, vol. 77, no. 8, pp. 813-819, 2012.

[10] H. Braak, K. Del Tredici, U. Rüb, R. A. I. de Vos, E. N. H. Jansen Steur, and E. Braak, "Staging of brain pathology related to sporadic Parkinson's disease," Neurobiology of Aging, vol. 24, no. 2, pp. 197-211, 2003.

[11] T. Jubault, S. M. Brambati, C. Degroot et al., "Regional brain stem atrophy in idiopathic Parkinson's disease detected by anatomical MRI," PloS one, vol. 4, no. 12, Article ID e8247, 2009.

[12] G. A. Korbel, G. Lalic, and M. D. Shair, "Reaction microarrays: a method for rapidly determining the enantiomeric excess of thousands of samples [16]," Journal of the American Chemical Society, vol. 123, no. 2, pp. 361-362, 2001.

[13] S. Mandel, E. Grünblatt, G. Maor, and M. B. H. Youdim, "Early and late gene changes in MPTP mice model of Parkinson's disease employing cDNA microarray," Neurochemical Research, vol. 27, no. 10, pp. 1231-1243, 2002.

[14] F. Simunovic, M. Yi, Y. Wang et al., "Gene expression profiling of substantia nigra dopamine neurons: further insights into Parkinson's disease pathology," Brain, vol. 132, no. 7, pp. 17951809, 2009.
[15] N. M. Lewandowski, S. Ju, M. Verbitsky et al., "Polyamine pathway contributes to the pathogenesis of Parkinson disease," Proceedings of the National Academy of Sciences of the United States of America, vol. 107, no. 39, pp. 16970-16975, 2010.

[16] R. Edgar, M. Domrachev, and A. E. Lash, "Gene Expression Omnibus: NCBI gene expression and hybridization array data repository," Nucleic Acids Research, vol. 30, no. 1, pp. 207-210, 2002.

[17] O. Troyanskaya, M. Cantor, G. Sherlock et al., "Missing value estimation methods for DNA microarrays," Bioinformatics, vol. 17, no. 6, pp. 520-525, 2001.

[18] A. Fujita, J. R. Sato, L. de Oliveira Rodrigues, C. E. Ferreira, and M. C. Sogayar, "Evaluating different methods of microarray data normalization," BMC Bioinformatics, vol. 7, no. 1, p. 469, 2006.

[19] G. K. Smyth, Limma: Linear Models For Microarray Data, in BioinFormatics and Computational Biology Solutions Using $R$ and Bioconductor, Springer, 2005.

[20] D. W. Huang, B. T. Sherman, and R. A. Lempicki, "Systematic and integrative analysis of large gene lists using DAVID bioinformatics resources," Nature Protocols, vol. 4, no. 1, pp. 44-57, 2009.

[21] B. Zhang, S. Kirov, and J. Snoddy, "WebGestalt: an integrated system for exploring gene sets in various biological contexts," Nucleic Acids Research, vol. 33, supplement 2, pp. W741-W748, 2005.

[22] D. Duncan, N. Prodduturi, and B. Zhang, "WebGestalt2: an updated and expanded version of the web-based gene set analysis toolkit," BMC Bioinformatics, vol. 11, supplement 4, p. $10,2010$.

[23] Y. Benjamini and Y. Hochberg, "Controlling the false discovery rate: a practical and powerful approach to multiple testing," Journal of the Royal Statistical Society B, vol. 57, pp. 289-300, 1995.

[24] R. E. Burke and N. Kholodilov, "Programmed cell death: does it play a role in Parkinson's disease?" Annals of Neurology, vol. 44, supplement 3, pp. S126-S133, 1998.

[25] M. P. Mattson, "Apoptosis in neurodegenerative disorders," Nature Reviews Molecular Cell Biology, vol. 1, no. 2, pp. 120-130, 2000.

[26] X. Cui, J. J. McGrath, T. H. J. Burne, A. Mackay-Sim, and D. W. Eyles, "Maternal vitamin D depletion alters neurogenesis in the developing rat brain," International Journal of Developmental Neuroscience, vol. 25, no. 4, pp. 227-232, 2007.

[27] H. L. Newmark and J. Newmark, "Vitamin D and Parkinson's disease-a hypothesis," Movement Disorders, vol. 22, no. 4, pp. 461-468, 2007.

[28] J. P. Kesby, X. Cui, P. Ko, J. J. McGrath, T. H. J. Burne, and D. W. Eyles, "Developmental vitamin D deficiency alters dopamine turnover in neonatal rat forebrain," Neuroscience Letters, vol. 461, no. 2, pp. 155-158, 2009.

[29] E. Garcion, N. Wion-Barbot, C. N. Montero-Menei, F. Berger, and D. Wion, "New clues about vitamin D functions in the nervous system," Trends in Endocrinology and Metabolism, vol. 13, no. 3, pp. 100-105, 2002.

[30] M. W. Butler, A. Burt, T. L. Edwards et al., "Vitamin D Receptor Gene as a Candidate Gene for Parkinson Disease," Annals of Human Genetics, vol. 75, no. 2, pp. 201-210, 2011.

[31] Z. Lv, "Association study between vitamin d receptor gene polymorphisms and patients with Parkinson disease in Chinese Han population," International Journal of Neuroscience, vol. 123, no. 1, pp. 60-64, 2012. 
[32] J.-S. Kim, Y.-I. Kim, C. Song et al., "Association of vitamin $\mathrm{D}$ receptor gene polymorphism and Parkinson's disease in Koreans," Journal of Korean Medical Science, vol. 20, no. 3, pp. 495-498, 2005.

[33] S. T. Lim, M. Airavaara, and B. K. Harvey, "Viral vectors for neurotrophic factor delivery: a gene therapy approach for neurodegenerative diseases of the CNS," Pharmacological Research, vol. 61, no. 1, pp. 14-26, 2010.

[34] J. R. Evans and R. A. Barker, "Neurotrophic factors as a therapeutic target for Parkinson's disease," Expert Opinion on Therapeutic Targets, vol. 12, no. 4, pp. 437-447, 2008.

[35] T. Kano, Y. Suzuki, M. Shibuya, K. Kiuchi, and M. Hagiwara, "Cocaine-induced CREB phosphorylation and c-Fos expression are suppressed in Parkinsonism model mice," NeuroReport, vol. 6, no. 16, pp. 2197-2200, 1995.

[36] A. D. Ebert, A. J. Beres, A. E. Barber, and C. N. Svendsen, "Human neural progenitor cells over-expressing IGF-1 protect dopamine neurons and restore function in a rat model of Parkinson's disease," Experimental Neurology, vol. 209, no. 1, pp. 213-223, 2008.

[37] R. Roshan, T. Ghosh, V. Scaria, and B. Pillai, "MicroRNAs: novel therapeutic targets in neurodegenerative diseases," Drug Discovery Today, vol. 14, no. 23-24, pp. 1123-1129, 2009.

[38] J. Xiong, "Emerging roles of microRNA-22 in human disease and normal physiology," Current Molecular Medicine, vol. 12, no. 3, pp. 247-258, 2012.

[39] R. Margis, R. Margis, and C. R. Rieder, "Identification of blood microRNAs associated to Parkinsonós disease," Journal of Biotechnology, vol. 152, no. 3, pp. 96-101, 2011.

[40] S. Altamura and M. U. Muckenthaler, "Iron toxicity in diseases of aging: alzheimer's disease, Parkinson's disease and atherosclerosis," Journal of Alzheimer's Disease, vol. 16, no. 4, pp. 879-895, 2009.

[41] M. Thomas and J. Jankovic, "Neurodegenerative disease and iron storage in the brain," Current Opinion in Neurology, vol. 17, no. 4, pp. 437-442, 2004.

[42] A. Khanna, S. Muthusamy, R. Liang, H. Sarojini, and E. Wang, "Gain of survival signaling by down-regulation of three key miRNAs in brain of calorie-restricted mice," Aging, vol. 3, no. 3, pp. 223-236, 2011.

[43] J. M. Timmins, L. Ozcan, T. A. Seimon et al., "Calcium/ calmodulin-dependent protein kinase II links ER stress with Fas and mitochondrial apoptosis pathways," Journal of Clinical Investigation, vol. 119, no. 10, pp. 2925-2941, 2009.

[44] M. E. Kalaitzakis, M. B. Graeber, S. M. Gentleman, and R. K. B. Pearce, "The dorsal motor nucleus of the vagus is not an obligatory trigger site of Parkinson's disease: a critical analysis of $\alpha$-synuclein staging," Neuropathology and Applied Neurobiology, vol. 34, no. 3, pp. 284-295, 2008.

[45] Louis, E. D. Babij R, E. Cortés, J. P. Vonsattel, and P. L. Faust, "The inferior olivary nucleus: a postmortem study of essential tremor cases versus controls," Movement Disorders, vol. 28, no. 6, pp. 779-786, 2013. 

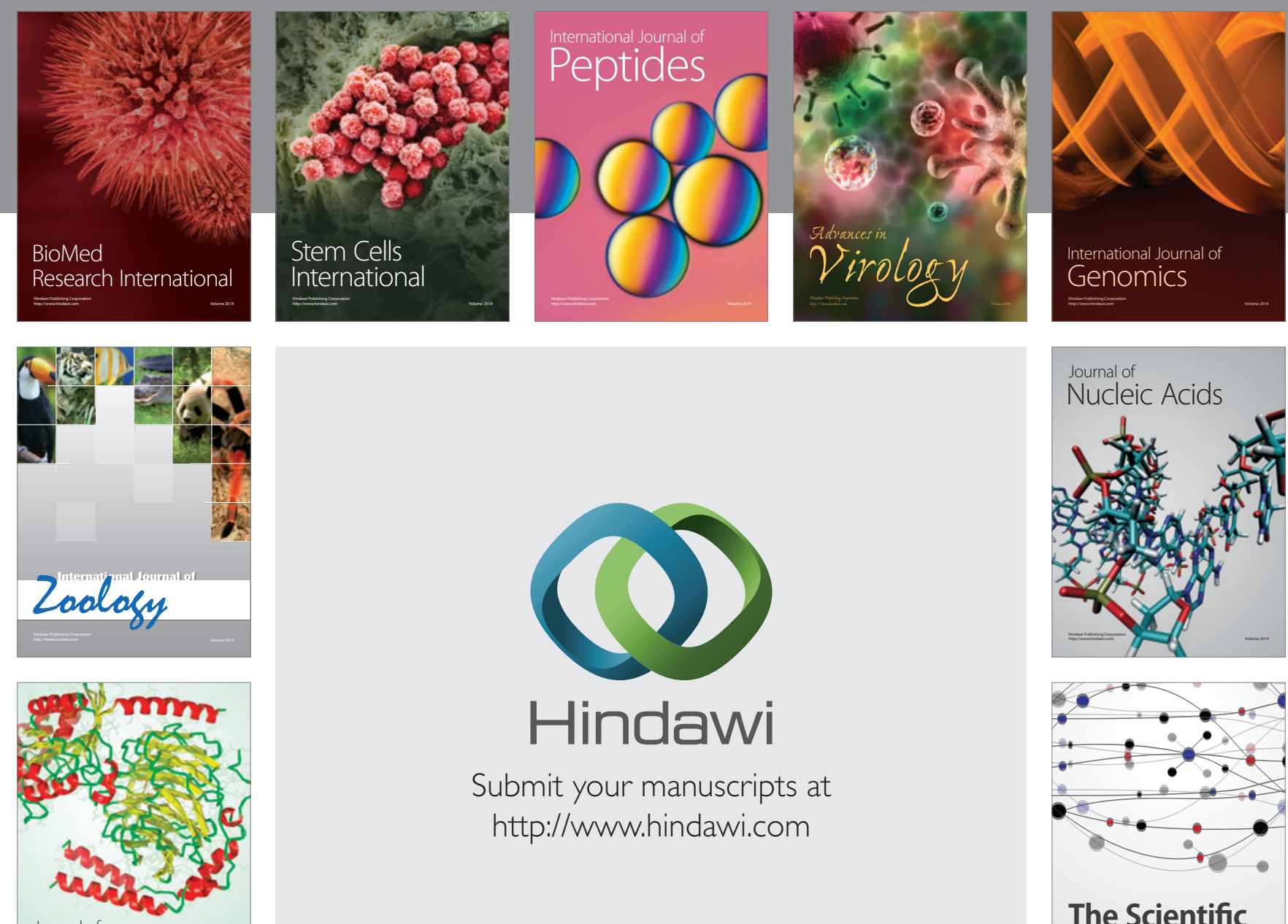

Submit your manuscripts at

http://www.hindawi.com

Journal of
Signal Transduction
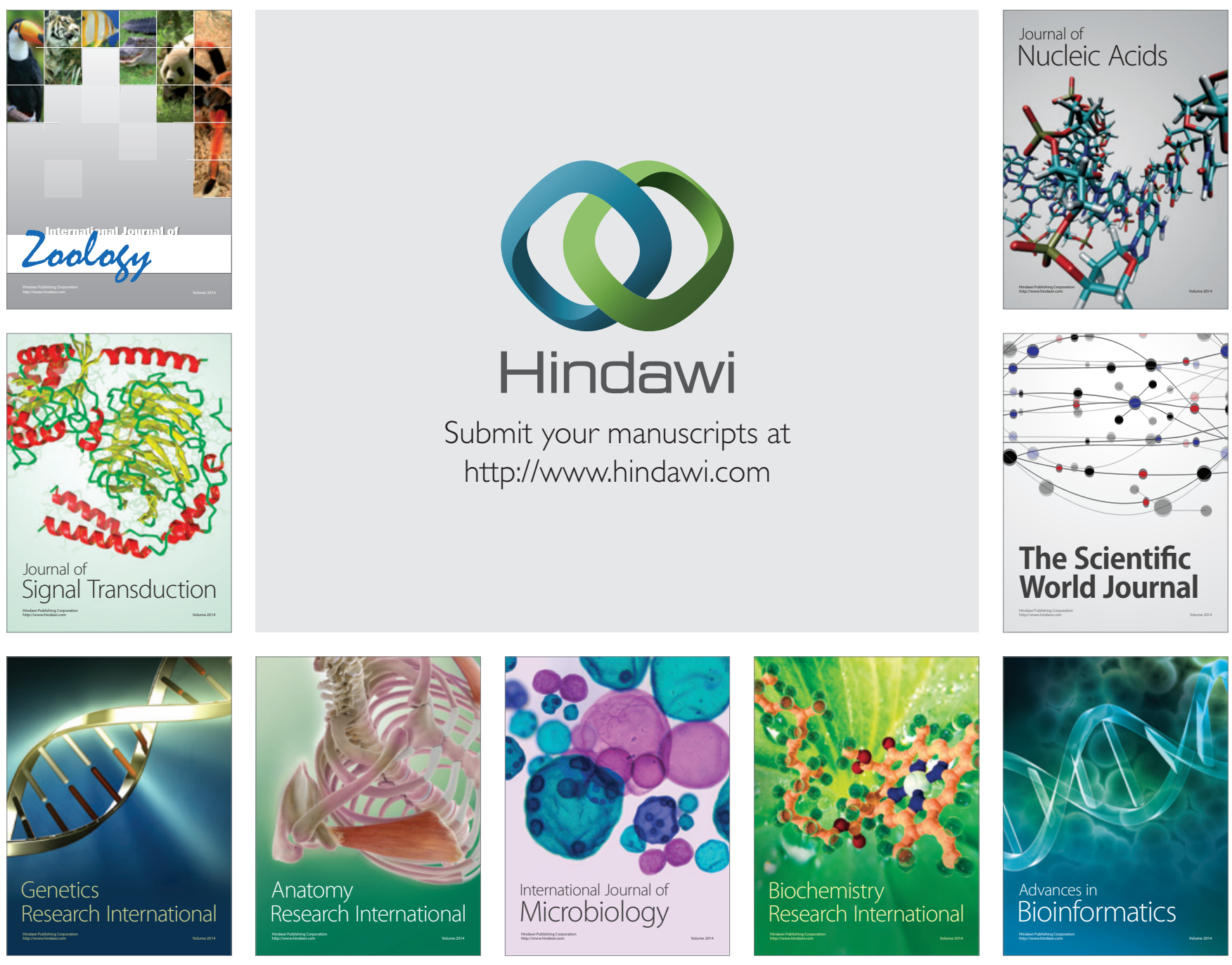

The Scientific World Journal
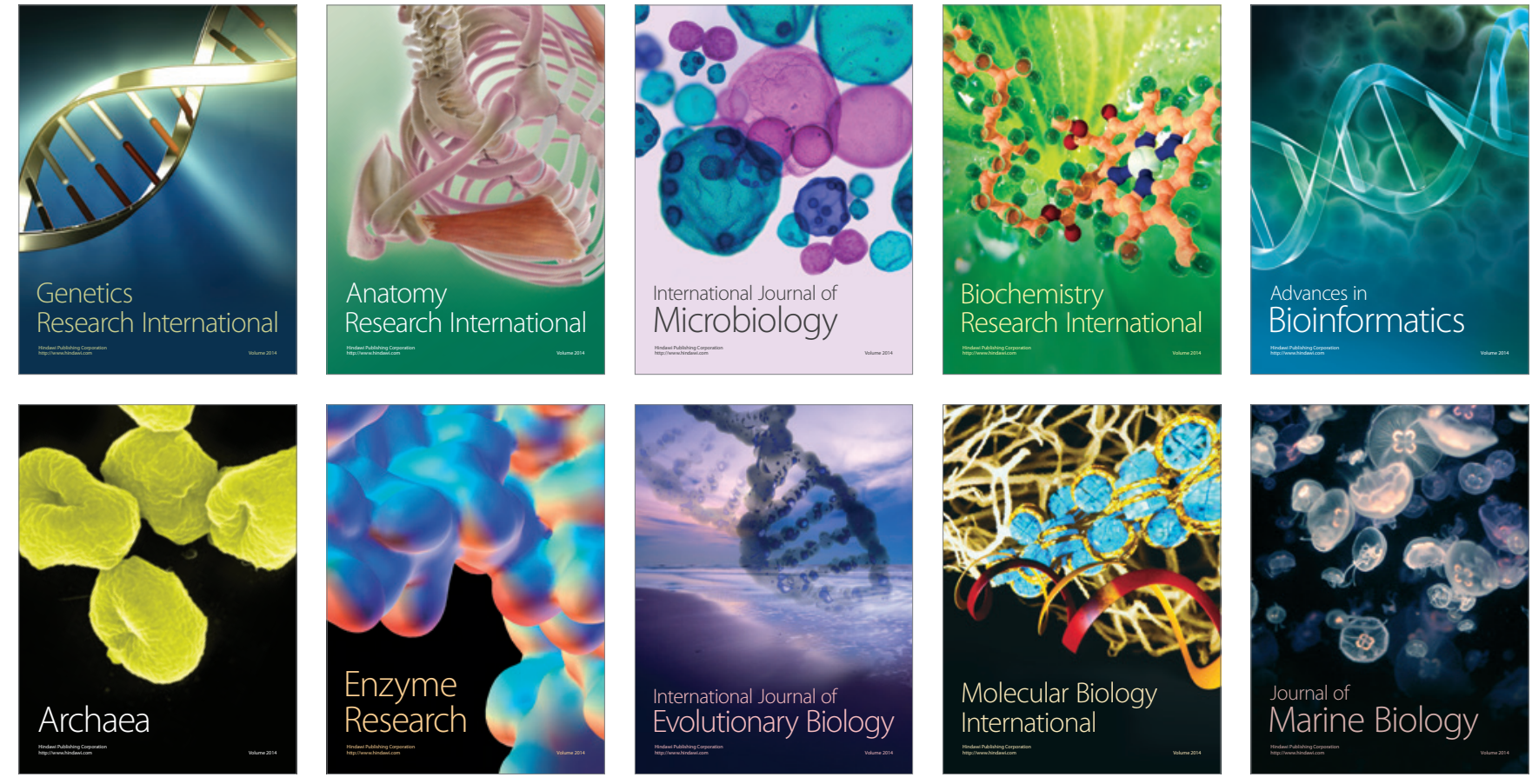